\title{
Study on the Technology and Properties of 3D Bioprinting SF/GT/n-HA Composite Scaffolds
}

\author{
Xiaofang $\mathrm{Wu}^{1}$, Kai Chen ${ }^{2 *}$, Dekun Zhang ${ }^{2 *}$, Linmin $\mathrm{Xu}^{2}$, Xuehui Yang ${ }^{3}$ \\ (1. School of Mechanical and Electrical Engineering, China University of Mining and Technology, Xuzhou 221116, \\ China ; \\ 2. School of Mechatronic Engineering, China University of Mining and Technology, Xuzhou 221116, China; \\ 3. Department of Mechanical Engineering, Purdue School of Engineering and Technology, IUPUI, Indianapolis,
} IN-46202, USA)

\begin{abstract}
In this paper, three kinds of natural polymer materials, silk fibroin (SF), gelatin (GT), and nano-hydroxyapatite (n-HA), are mixed as 3D printing bioink to mimic protein polysaccharide and collagen fibers in natural articular cartilage. By changing the SF content, SF/GT/n-HA composite scaffolds with different ratios are prepared using 3D bioprinting technology. The microstructure and morphology, biological properties and mechanical properties of composite scaffolds are characterized. The results show that the printing precision of the bioink with $10 \% \mathrm{SF}$ is best, and the composite scaffold with $10 \% \mathrm{SF}$ also exhibits better mechanical properties, whose tensile elastic modulus is $10.60 \pm 0.32 \mathrm{MPa}$ and the compression elastic modulus is $1.22 \pm 0.06 \mathrm{MPa}$. These studies are helpful to understand the interaction between SF, GT and n-HA, and provide a theoretical basis for the preparation of better silk fibroin-based composite scaffolds.
\end{abstract}

Keywords: 3D bioprinting; bioink; silk fibroin; composite scaffolds

\section{Introduction}

Tissue engineering technology is expected to achieve articular cartilage repair, but the ideal cartilage tissue engineering scaffold is still a great challenge for researchers [1]. The emerging 3D bioprinting technology can macroscopically regulate the number, size and distribution of stomata in scaffolds, and the key to 3D bioprinting is the selection of bioink [2]. Silk fibroin (SF) and gelatin (GT) are two typical natural polymer materials which are widely used in biological field due to their biocompatibility, degradability and low immunogenicity [3-4]. Nano-hydroxyapatite (n-HA) is the main inorganic component of bone mineral matrix, which can improve bone integration and promote bone cells proliferation. Despite much research on silk fibroin-based bioscaffolds [5], most of them are made by traditional methods [6-7].

*Corresponding author: Kai Chen, Dekun Zhang

E-mail address: cumtck@cumt.edu.cn, dkzhang@cumt.edu.cn

This is the author's manuscript of the article published in final edited form as:

Wu, X., Chen, K., Zhang, D., Xu, L., \& Yang, X. (2019). Study on the technology and properties of 3D bioprinting SF/GT/n-HA composite scaffolds. Materials Letters, 238, 89-92. https://doi.org/10.1016/j.matlet.2018.11.151 
This paper adopts 3D bioprinting technology, and uses SF, GT and n-HA as the bioink to prepare and study SF/GT/n-HA composite scaffolds. The advantage of the bioink is that it not only can mimic the proteoglycan and collagen fibers in natural cartilage [8], but also can avoid the blockage of needles in the $3 \mathrm{D}$ printing process by blending silk and gelatin solutions, thus ensuring the homogeneity of silk deposition and the precision of the printed structure.

\section{Experiment}

\subsection{Experimental Materials}

Soluble lyophilized silk protein was purchased from Suzhou Simet Biotechnology Company. Gelatin was provided by Shanghai Maclean Biochemical Technology Co., Ltd., adhesive strength is $-250 \mathrm{~g}$ Bloom. Nano-hydroxyapatite, element content $\mathrm{Ca}_{10}\left(\mathrm{PO}_{4}\right)_{6}(\mathrm{OH})_{2} \geq 99.5 \%$. Well-behaved SBF simulated body fluid was provided by Xi'an Haote Biological Co., Ltd.

\subsection{Preparation of bioink}

SF $(0 \%, 6 \%, 8 \%, 10 \%, 12 \%)$, GT $(10 \%)$ and n-HA (3\%) are weighed according to different mass fractions, and dissolved in deionized water to prepare five kinds of SF/GT/n-HA composite hydrogel precursors, which are used as 3D printing bioink and stored in a refrigerator at $4{ }^{\circ} \mathrm{C}$.

\subsection{D printing}

The prepared hydrogel bioink was printed using a 3D printing system (Regenovo 3D Bioprinter V2.0, Regenovo Biotechnology Co., Ltd). The parameters of the 3D printing were adjusted after the finding that the bioink with $12 \%$ SF could not print out filaments using the same needle diameter. The ratio was discarded in the subsequent experiments. Tab.1 shows the bioprinting parameters for the other concentration ratios.

Tab.1 3D printing parameters of SF/GT/n-HA composite scaffolds with different ratios

\begin{tabular}{ccccccc}
\hline $\begin{array}{c}\text { SF } \\
\text { content } \\
(\mathrm{wt} \%)\end{array}$ & $\begin{array}{c}\text { Printing } \\
\text { pressure } \\
(\mathrm{MPa})\end{array}$ & $\begin{array}{c}\text { Barrel } \\
\text { temperature } \\
\left({ }^{\circ} \mathrm{C}\right)\end{array}$ & $\begin{array}{c}\text { Needle } \\
\text { diameter } \\
(\mathrm{mm})\end{array}$ & $\begin{array}{c}\text { Printing } \\
\text { rate } \\
(\mathrm{mm} / \mathrm{s})\end{array}$ & $\begin{array}{c}\text { Printing } \\
\text { spacing } \\
(\mathrm{mm})\end{array}$ & $\begin{array}{c}\text { Platform } \\
\text { temperature } \\
\left({ }^{\circ} \mathrm{C}\right)\end{array}$ \\
\hline 0 & 0.20 & 28 & 0.26 & 6 & 0.7 & 5 \\
6 & 0.31 & 30 & 0.26 & 6 & 0.7 & 5 \\
8 & 0.30 & 30 & 0.26 & 6 & 0.7 & 5 \\
10 & 0.19 & 30 & 0.26 & 6 & 0.7 & 5 \\
\hline
\end{tabular}




\subsection{Characterization}

The microstructure of samples is characterized by X-ray diffraction (XRD) (Bruker D8 ADVANCE, Germany) and Fourier transforms infrared spectroscopy (FTIR) (Bruker VTRTEX $80 \mathrm{~V}$, Germany). The morphology is characterized by Scanning electron microscope (SEM) (Quanta250, FEI, USA). The biological properties are characterized by swelling (immersed in deionized water), degradability (immersed in SBF) and hydrophilicity, among which the hydrophilicity is evaluated by the wetting angle measured by a contact angle tester. The tension and compression properties are tested using the electronic universal testing machine (WDW-2, Hengxu, China). The rheological properties are evaluated by a rheometer (DHR-2 TA, USA).

\section{Results and discussion}

3.1 3D printing SF/GT/n-HA composite hydrogel scaffolds

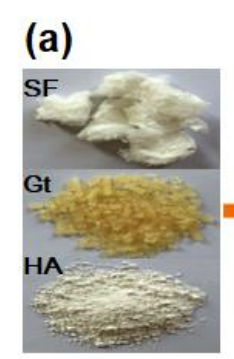

(b)

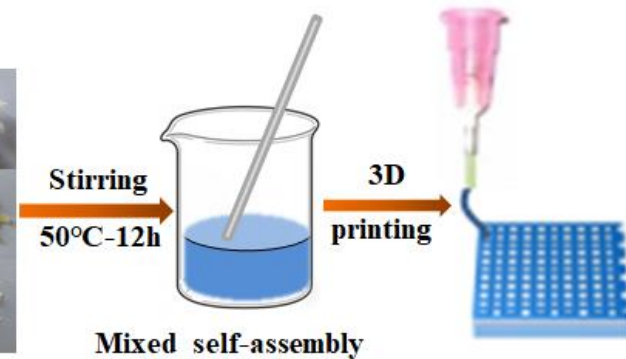

(c)

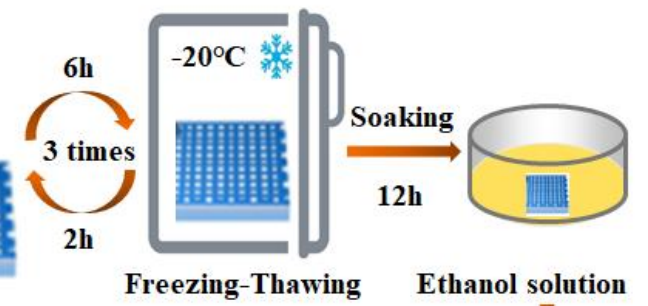

Freezing-Thawing
Ethanol solution

(d)

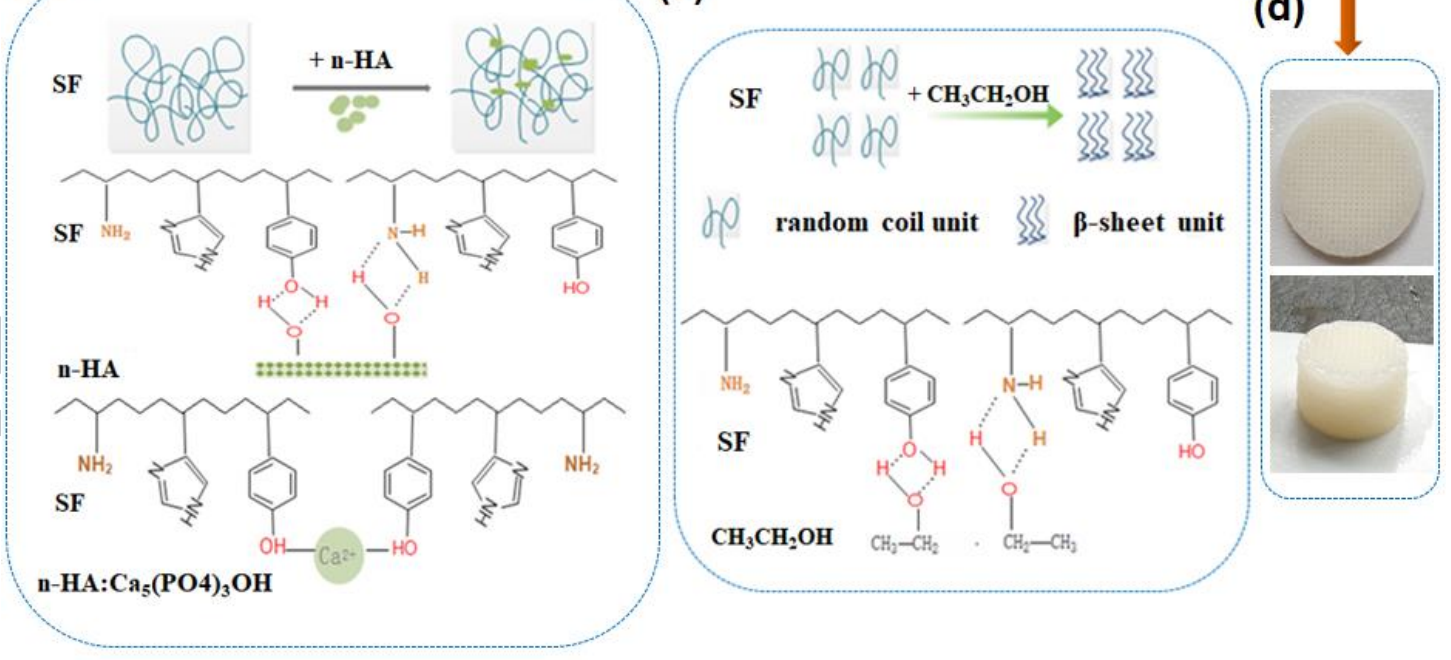

Fig.1. (a) The preparation process of 3D printing SF/GT/n-HA composite scaffolds;

The reaction mechanism of (b) SF and n-HA (c) SF and ethanol; (d) 3D printed samples with $10 \% \mathrm{SF}$

The prepared bioink is loaded into the barrel of the 3D printer and square, circular, strip and cylindrical samples are printed separately. It is found that the filaments out from the bioink containing $10 \% \mathrm{SF}$ are homogenized during the printing process, and the printed structure is able 
to maintain good precision. Subsequently, the samples are frozen for 12 hours in a cryogenic storage tank at $-20^{\circ} \mathrm{C}$, then taken out and thawed at room temperature for 2 hours, repeating the above process 3-4 times. Finally, the samples are soaked in absolute ethanol for storage. The preparation process and reaction mechanisms are shown in Fig. 1.

\subsection{Microstructure and morphology analysis}

As shown in Fig.2(a), the diffraction peaks of n-HA $\left(2 \theta\right.$ is $\left.31.8^{\circ}, 32.0^{\circ}, 32.9^{\circ}\right)$ become sharper with the increase of SF concentration, indicating that the addition of SF can induce the mineralization and crystallization of n-HA [6]. Mainly because of chemical bonding between $\mathrm{Ca}^{2+}$ ions of n-HA and the -OH group of SF, n-HA deposits in the form of crystals which promote the formation of normal bone.

As shown in Fig.2(b), the absorption peak pattern of the $-\mathrm{NH}_{2}$ group at $3287 \mathrm{~cm}^{-1}$ widens gradually; the characteristic peak of the free $-\mathrm{OH}$ group at $3420 \mathrm{~cm}^{-1}$ in pure n-HA disappears; the absorption peak intensity of amide I band $\left(1631 \mathrm{~cm}^{-1}\right)$, amide II band $\left(1545 \mathrm{~cm}^{-1}\right)$ and amide III band $\left(1237 \mathrm{~cm}^{-1}\right)$ gradually strengthen with the increasing of SF concentration.

(a)

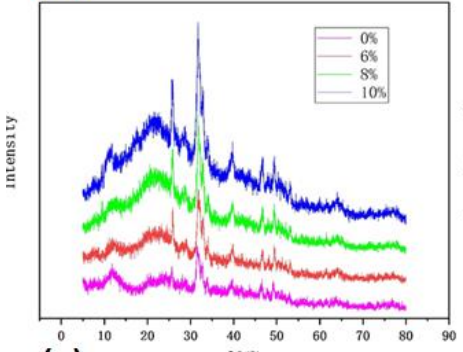

(c)

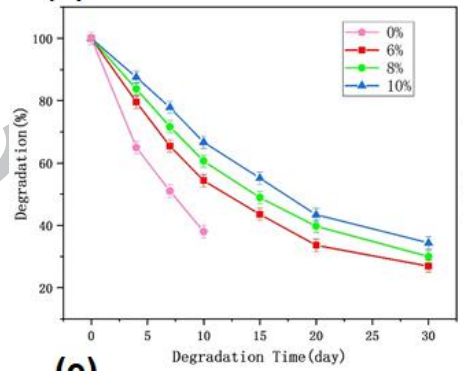

(e)

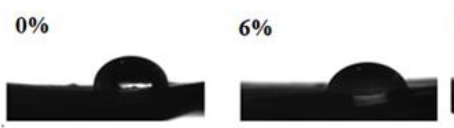

(b)

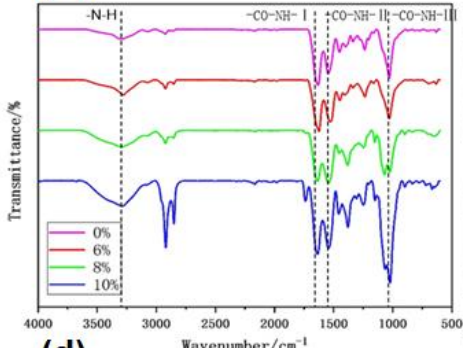

(d)

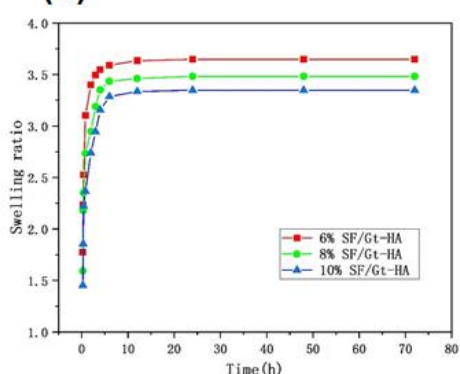

$8 \%$ (f)

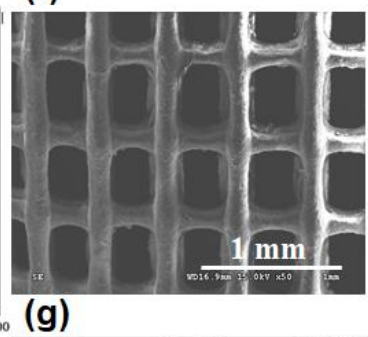

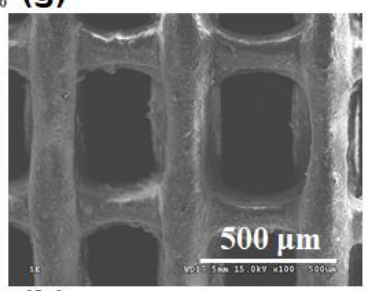

(h)

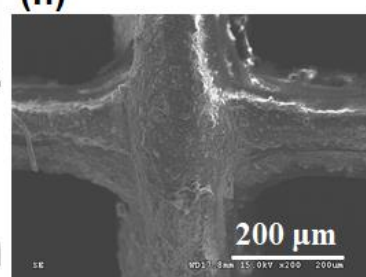

Fig.2(a)XRD pattern of samples with $0 \% \mathrm{SF}, 6 \% \mathrm{SF}, 8 \% \mathrm{SF}, 10 \% \mathrm{SF}$; (b)FTIR spectrum; (c)Degradability curves; (d)Swelling rate curves; (e)Hydrophilic test; (f)SEM 50x; (g)SEM 100x; (h)SEM 150x

Fig.2(f)(g)(h) show scanning electron micrographs of the sample with $10 \%$ SF at different magnifications. The 3D printed SF/GT/n-HA composite scaffold exhibits a dense 
three-dimensional network structure with smooth pore wall, uniform pore size and excellent interlayer bonding. While the filament diameter shrinks slightly, this may be caused by the moisture evaporated from multiple freezing and thawing [9].

\subsection{Biological properties analysis}

According to Fig.2(c), the degradation rate of the $0 \%$ SF sample is fastest, and the structure is loose and silky like floc on the 7 th day; the degradation rate of the $10 \%$ SF sample is slowest. This indicates that the addition of SF improves the degradability of samples. With the SF content increasing, the number of hydrogen bonds formed among molecules increases and the crosslinking degree among segments enlarges, so the binding force is enhanced and the degradability is lowered.

According to Fig.2(d), the larger the SF content, the worse is the swelling property. The main reason is that with the increase of $\mathrm{SF}$ content, the hydrophilic $-\mathrm{NH}_{2}$ group is easily forms hydrogen bonds with the -OH group of ethanol, so the hydrophilic groups are destroyed and the hydrophobic fragments are rearranged into highly crystalline ordered $\beta$-sheet structure, which decreases the space for accommodating water molecules.

According to Fig.2(e), the $10 \%$ SF sample displays the smallest contact angle and the strongest hydrophilicity, while the $0 \% \mathrm{SF}$ sample is opposite. It is possible that SF surface contains hydrophilic $-\mathrm{NH}_{2}$ groups, the higher the $\mathrm{SF}$ content, the more hydrophilic $-\mathrm{NH}_{2}$ groups, and the better materials hydrophilicity.

\subsection{Mechanical properties analysis}

According to Fig.3(a)(b), the tensile elastic modulus of the $6 \%, 8 \%$ and $10 \%$ samples is $2.53 \pm 0.16 \mathrm{Mpa}, 6.09 \pm 0.18 \mathrm{Mpa}$ and $10.60 \pm 0.32 \mathrm{Mpa}$, respectively. The compressive elastic modulus is $0.45 \pm 0.02 \mathrm{Mpa}, 0.60 \pm 0.03 \mathrm{Mpa}$ and $1.22 \pm 0.06 \mathrm{Mpa}$, respectively. Furthermore, the elastic moduli of the samples increase with an increase of SF concentration. This can be attributed to an increase in the number of hydrogen bonds between SF and n-HA or ethanol molecules, leading to the rearrangement of peptide chains, thus transforming SF from a random coiled SilkI structure to an ordered crystallized SilkII structure, which helps to enhance the intermolecular interaction and improve the deformability of silk fibroin.

Fig.3(c)(d) show, when the strain is $0.01 \% \sim 1 \%$, the storage modulus $\left(\mathrm{G}^{\prime}\right)$ of the composite 
scaffolds is much larger than the loss modulus $\left(\mathrm{G}^{\prime \prime}\right)$, and both values are relatively stable, so the loss angle $(\delta)$ is basically the same; when the strain continues to increase, the $\mathrm{G}^{\prime}$ of each sample decreases dramatically, and that of the $6 \%$ sample decreases most obviously, that is why the $\delta$ increases rapidly. This may be due to that the $6 \%$ samples possess the lowest cross-linking density and the weakest inter chain binding force, so they start sliding most easily under the external forces, resulting in structural damage.

(a)

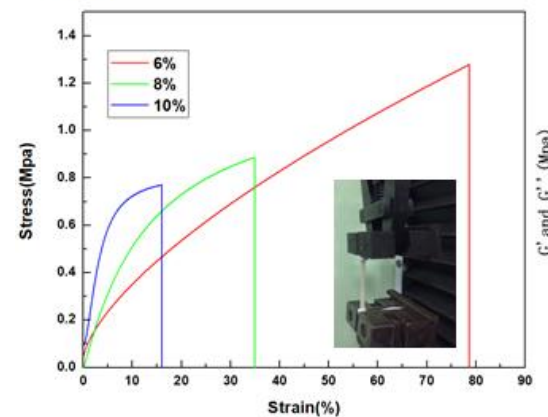

(b)

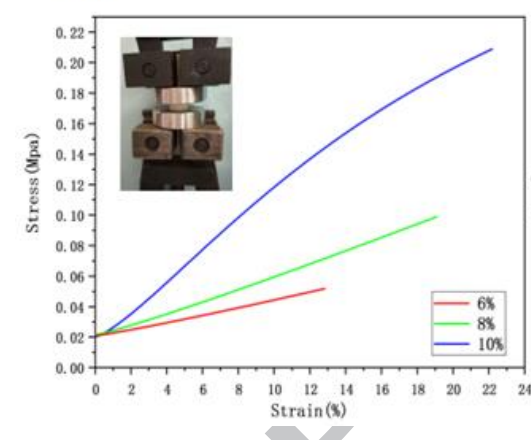

(c)

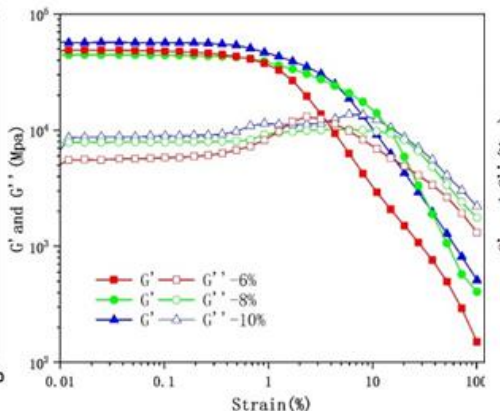

(d)

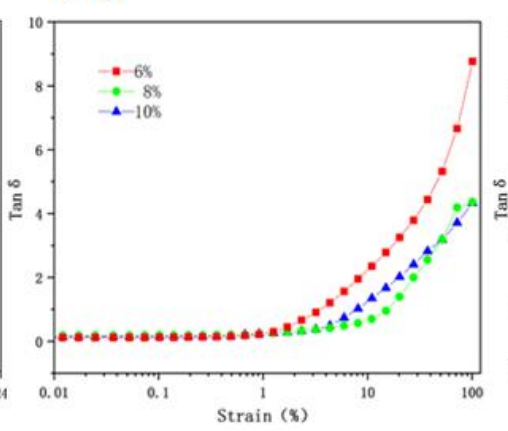

(e)

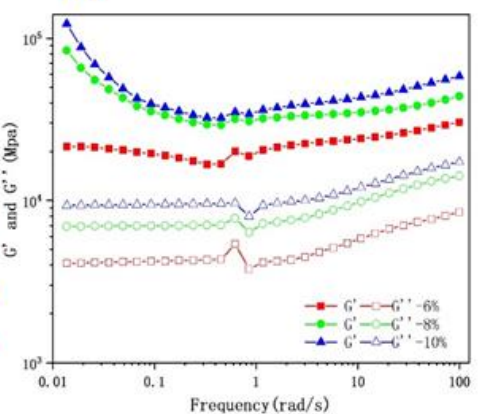

(f)

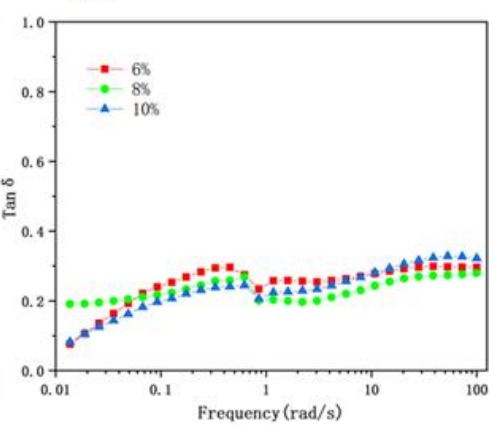

Fig.3.(a)Tensile stress-strain curves; (b)Compressive stress-strain curves;(c)Curves of G' and G" with strain;(d)Curves of $\delta$ with strain;(e)Curves of G' and G" with frequency;(f)Curves of $\delta$ with frequency

Fig.3(e)(f) illustrate the $G^{\prime}$ and $G^{\prime \prime}$ of the composite scaffolds fluctuate slightly with frequency, and the whole presents a slow increasing trend after the frequency reaching $1 \mathrm{rad} / \mathrm{s}$. The $\delta$ increases gradually as the frequency is lower than $1 \mathrm{rad} / \mathrm{s}$, and fluctuates very little when the frequency reaches more than $1 \mathrm{rad} / \mathrm{s}$. On the whole, the SF/GT/n-HA composite scaffolds still maintain an orderly structure at the frequency of $0.01 \sim 100$. The loss angle is slightly larger than that of natural cartilage, which may be due to the relatively high viscosity of SF and GT under wet conditions. Therefore, the content of n-HA can be appropriately increased to reduce the viscosity.

\section{Conclusion}

SF/GT/n-HA composite scaffolds with different SF contents were prepared by using 3D bioprinting technology. According to the microstructure and morphology analysis, the bioink with 
$10 \%$ SF displays the best printing performance, and the printed composite scaffolds exhibit complete structures and uniform pores. According to biological performance analysis, the increase of SF content can improve the hydrophilicity, slow down the biodegradation rate, and reduce the swelling ratio. According to mechanical properties analysis, the scaffolds with $10 \%$ SF possess better mechanical strength. Therefore, the SF/GT/n-HA composite hydrogel is expected to be an ideal biomaterial for 3D bioprinting technology to prepare silk fibroin based composite scaffolds.

\section{Acknowledgements}

This research is supported by Fundamental Research Funds for the Central Universities (2017XKZD08) and Priority Academic Program Development of Jiangsu Higher Education Institutions (PAPD).

\section{References}

[1] Shi W, Sun M, Hu X, et al. Structurally and functionally optimized silk- fibroin-gelatin scaffold using 3D printing to repair cartilage injury in vitro and in vivo[J]. Advanced Materials, 2017, 29(29): 1701089.

[2] Li L, Zhu Y, Yang J. 3D bioprinting of cellulose with controlled porous structures from NMMO[J]. Materials Letters, 2018, 210:136-138.

[3] Das S, Pati F, Chameettachal S, et al. Enhanced redifferentiation of chondrocytes on microperiodic silk/gelatin scaffolds: toward tailor-made tissue engineering[J]. Biomacromolecules, 2013, 14(2): 311-321.

[4] Lu Q, Feng Q, Hu K, et al. Preparation of three-dimensional fibroin/collagen scaffolds in various $\mathrm{pH}$ conditions[J]. Journal of Materials Science: Materials in Medicine, 2008, 19(2): 629-634.

[5] Mottaghitalab F, Hosseinkhani H, Shokrgozar M A, et al. Silk as a potential candidate for bone tissue engineering[J]. Journal of Controlled Release, 2015, 215: 112-128.

[6] Kim M H, Kim B S, Lee J, et al. Silk fibroin/hydroxyapatite composite hydrogel induced by gamma-ray irradiation for bone tissue engineering[J]. Biomaterials research, 2017, 21(1): 12.

[7] Ming J, Jiang Z, Wang P, et al. Silk fibroin/sodium alginate fibrous hydrogels regulated hydroxyapatite crystal growth[J]. Materials Science and Engineering: C, 2015, 51:287-293.

[8] Zhou Y, Liang K, Zhao S, et al. Photopolymerized maleilated chitosan/methacrylated silk 
fibroin micro/nanocomposite hydrogels as potential scaffolds for cartilage tissue engineering[J]. International journal of biological macromolecules, 2018, 108: 383-390.

[9] Chen K, Liu J, Yang X, et al. Preparation, optimization and property of PVA-HA/PAA composite hydrogel[J]. Materials Science and Engineering: C, 2017, 78: 520-529. 


\section{Highlights:}

1. SF, GT and n-HA is constructed as 3D printing bioink.

2. SF/GT/n-HA composite scaffolds are prepared by 3D printing technology.

3. Scaffolds have complete structure and excellent performance. 